This is an electronic reprint of the original article. This reprint may differ from the original in pagination and typographic detail.

Author(s): Helfenstein, Sacha; Kaikova, Olena; Khriyenko, Oleksiy; Terziyan, Vagan

Title: $\quad$ Emotional Business Intelligence : Enabling experience-centric business with the FeelingsExplorer

Year: $\quad 2014$

Version:

Please cite the original version:

Helfenstein, S., Kaikova, O., Khriyenko, O., \& Terziyan, V. (2014). Emotional Business Intelligence : Enabling experience-centric business with the FeelingsExplorer. In L. Gomes, J. Wtorek, A. Costa, H. Sawada, M. Manic, \& P. Strumillo (Eds.), Proceedings of the 7th International Conference on Human System Interactions (HSI) (pp. 14-21). Institute of Electrical and Electronic Engineers (IEEE).

https://doi.org/10.1109/HSI.2014.6860441

All material supplied via JYX is protected by copyright and other intellectual property rights, and duplication or sale of all or part of any of the repository collections is not permitted, except that material may be duplicated by you for your research use or educational purposes in electronic or print form. You must obtain permission for any other use. Electronic or print copies may not be offered, whether for sale or otherwise to anyone who is not an authorised user. 


\title{
Emotional Business Intelligence
}

\author{
Enabling experience-centric business with the FeelingsExplorer
}

\author{
Sacha Helfenstein ${ }^{1}$, Olena Kaikova ${ }^{2}$, Oleksiy Khriyenko ${ }^{2}$, Vagan Terziyan ${ }^{2}$ \\ ${ }^{1}$ Agora Center, ${ }^{2}$ Department of Mathematical Information Technology \\ University of Jyväskylä \\ Jyväskylä, Finland \\ sacha.helfenstein@jyu.fi, olena.o.kaikova@jyu.fi, oleksiy.o.khriyenko@jyu.fi,vagan.terziyan@jyu.fi
}

\begin{abstract}
The domain of Emotional Business Intelligence (EBI) aims to support business-relevant emotional and emotionaware decisions in addition to rational decision making. EBI originates from three root domains: Emotional Business, Emotional Intelligence and Business Intelligence (BI). In this paper we emphasize emotional empowerment of the traditional BI function; outline its main characteristics as a business working model of an emotionally smart, continuously learning organization; and introduce a first candidate of the EBI Toolkit, the FeelingsExplorer (FE). FE is a mash-up browser based on $4 \mathrm{i}$ ("ForEye") technology, capable of visualizing objects in an emotional semantic space and thereby supporting decision making on emotional grounds. It takes metadata as input and visualizes the personalized "emotional similarity" of products, services, and customers. Different scenarios for FE application and overall implications of EBI for business and human technology are discussed.
\end{abstract}

Decision support systems, decision making, context-aware computing, affective computing, emotive data, Business Intelligence, Customer Experience

\section{INTRODUCTION}

Business organizations are complex systems that-for as long as they exist-have researched and rationalized about customers, in order to expose and elicit their needs and preferences, to scrutinize and sway their decisions and behavior, to design and deliver products and services of their desire, and finally to win and nurture their loyalty. In this sense, human- and customer centricity has long been part of the business culture and are common managerial jargon, yet, so we claim, we are to date at the brink of completely new, powerful approaches to support the attunement and intimacy between business and their human stakeholders. This forthcoming evolution will be driven by two interdependent domains: On the system- and technology-inspired side this pertains to the leveraging of massively evolving data collection and analytical capabilities for business-relevant decision making; and on the human-inspired side this regards a return, and finally breakthrough, of the emotion topic to complement and enhance current customer notions.

In this paper we introduce an important new businesscustomer relation paradigm titled Emotional Business Intelligence $(E B I)$, outline its relevance, and envisage one concrete technological enabler and impact example called FeelingsExplorer. At this stage in our research, our solution is mostly on Proof of Concept level and the presentation of evidence resembles therefore that of a "Gedankenexperiment" about future business system intelligence in dealing with its human dimensions.

Throughout history it is obvious that the homo economicus has been the dominating model for describing and explaining human and organizational behavior in commerce contexts. This homo economicus is rational and logical, cognizant and utilitarian, deterministic and computational, and therefore - and this is important - assumingly computable, predictable, and controllable. All of these attributes, alongside its fit to the popular human information processor model [1] [2], made the homo economicus seemingly an archetypical business companion, and an ideal inhabitant of the knowledge society in a digital age. Above all, the homo economicus is a very Business Intelligence (BI)-friendly notion, considering that BI (by providing analytical processing and practical presentation of business data) traditionally supports rational decision making [3] [4] [5] [6] [7].

However, humans in- and outside business organizations cannot be aptly described and understood in rational terms alone, and for this reason the homo economicus is a fallacious abstraction that undermines the mission of raising businesscustomer proximity as well as the validity and power of business-relevant decision-making models. This conclusion per se is not new, and has -implicitly and explicitlyaccompanied the discourse between economic and psychological takes on decision-making throughout history [8].

Here we claim that introducing emotions into the business equation is an opportunity to generate new value with customers and employees. And on top of this it is a necessity not only to enhance the naturalistic model validity but importantly also to deal with the future data load of BI. In this vein, we have recently started witnessing welcomed changes in how companies, products and services, as well as people's relation to these, are conceptualized. These changes have been driven by a rise of emotion-related themes, emphasizing on hedonic and symbolic product and service meanings, user and customer experiences (see e.g., [9]'s research agenda), and intangible organizational assets (e.g., [10]).

Yet, as popular as these changes have been with business evangelists, they have often proven difficult in practice. Our paper is a contribution to reinforce the valuable evolution towards experience-centricity by offering for it a conceptual handle and boosting the business practical relevance. The 
proposed conceptions intend to challenge incumbent business models in general, and at their core, they urge to review the functioning and aptness of BI as an organization's central data processing nervous system. To do so, we inquire about the role of experiences (particularly emotions) in business and outline the future of $\mathrm{BI}$ in this emerging emotional context, calling it EBI.

EBI results conceptually from fusing three major tenets: Emotional Business (EB) + Emotional Intelligence (EI) + Business Intelligence (BI). In this paper we emphasize the Emotional (Business Intelligence) dimension of $E B I$ and we introduce a kind of emotional browser (FeelingsExplorer). This tool is aimed at supporting processes of business- and consumption-related decision-making within an emotional context. The rest of the text is organized as follows: in Section II we argue the importance and expectations of EBI; in Section III lay out the main building blocks of EBI; in Section IV we present the FeelingsExplorer as a browser for EBI; and we summarize and conclude in Sections V and VI.

\section{Why EMOTIONAL BUSINESS INTELLIGENCE ?}

The intangibles theme pertaining to the business value of experiences and emotions has for a while been present in the business models and strategy discourse landscape and continues to be highly popular with trend-setting industry analysts and research institutes, such as Forrester, Gardner, Forbes, and Harvard Business Review. Like most of the propagated experience-centric initiatives, EBI aims to bring businesses closer to their customers, creating more meaningful products and services and adding brand value.

The modern academic discourse on the matter dates back to 1982 when Holbrook and Hirschman rallied for the reinclusion of experiences in consumer theory and business models [11]. Fifteen years later Pine and Gilmore proclaimed experiences as "the next step in ... the progression of economic value" [12]. And to date, another fifteen years later, the call is for ever new types of experience-centricity, pertaining to business and innovation in the age of meaning, particularly emotional meaning [13] [14] [15] [16].

On the business side, a lot of companies set out over the last decade to take this "experience-step", but many of them have stumbled. Customer Experience (CX) is a widely known concept denoting single and summed upl experiences a customer has with a supplier/provider of goods/services over the duration of their relationship. The associated business case states that the ability of a company to deliver good experience reflects in consumer spending with the company. However, despite many sophisticated CX management activities, there are certain aspects that to date remain challenging. Particularly emotions have proven a very elusive construct, and their application to business practice has largely remained confined to methods around emotionally considerate design and development of products and services (see e.g., [17] for Kansei engineering tradition), as well the use of affective elements in marketing and sales. This means, that business relevance of emotions have been recognized and instrumentalized, however, mainly within conventional business unit silos.
As a result, even topic-affine organizations and business leaders to date tend to miss implementation-critical elements, particularly when the ambition is to lead the company beyond the stage of one-off CX-projects or strategic lip services. These holdups concern: (1) factual proof and conviction on quantifiable Return-On-Investment ("is experience-centric business really worth it?"), (2) conceptual grasp and strategic blueprint ("what is experience-centric business all about?"), and (3) practical and operational implementation guidance ("how to industrialize experience-centric business?"). These are also the expectation benchmarks EBI should live up to, and therefore among its requirements is to serve as a key strategic constituent for an experience-centric business with tangible business-practical implications, the most important of which are measurability and steerability. However, before exhibiting the EBI concept in more detail let us still return to the question about why emotionality is so essential.

From a human point of view, the need for including experiences and emotions into the business equation is rather obvious: It is about treating people as people; within your own organization, your partner network, and your customer population. People experience the world through an emotional lens, make emotionally-inspired choices, behave in emotionladen ways, and base their thinking and future action in relevant ways on the emotional valence of past experiences. Emotions are phylo- and ontogenetically primary mental faculties and part of cognition at large, something [18] termed "ratiomorph" (see also [19]). Therefore, the often cited antithesis between cognition or ratio on the one side, and emotion on the other side is an inappropriate, if not to say deceptive, paradigm.

The affective influence on human thinking and behavior is especially pronounced in situations of heightened complexity, uncertainty, and urgency - rather typical situational attributes in business environments. Further, emotions play an important role in guiding human attention and reasoning towards aspects that are deemed as personally relevant and important, and they provide the carrier with an immediate valence-related appraisal of good versus bad (for him- or herself). This influence of emotions is particularly pertinent in the context of decisionmaking and intentional action [20], [21]. This means, intuition (in a certain way aggregated knowledge and experience) guides us to attend to personally significant matters, to make right choices, and towards meaningful actions. In fact, it is this aspect of information processing where the biggest differentiator between machines and humans lies, and with this also one of the biggest future challenges and opportunities for artificial reasoning especially in the context of Big Data.

According to [22], a major future Big Data dilemma states that more data means on the one hand, more potential to detect useful patterns for decision making, and on the other hand, reduced the likelihood for any data mining tool to discover these patterns within an acceptable time frame [23]. Similar to human intuition and emotion-based judgment, we argue for a decision-support tool that breaks out of rationality [24] and integrates the kind of wisdom and capability for preparing the emotionally inspired and intuitive decisions [25]. This would be definitely helpful for various business decisions on top of Big Data [26], assuming that such an emotional context will 
not only enhance the capabilities of available BI tools, but support business performance - internal and external - through more human valid choices.

\section{EBI: CONCEPTUAL FrAME AND BUSINESS MODEL}

\section{A. $E B I=E B+E I+B I$}

As indicated earlier, the vision and objectives of Emotional Business Intelligence (EBI) are derivative and integrative concepts building on key features and associated technologies of three underlying component domains:

1) Emotional Business (EB), [13] [14] [27], is about emotional awareness and the use of emotions to improve: (a) the effectiveness of a business organization in producing products and services through better managed internal organizational activity (e.g., emotionally excited employees); (b) the performance in selling products and services through better organized customer motivation and satisfaction (evoking long-lasting customer passion); and (c) the flexibility of business models by linking the functional aspects (product or service) to emotional aspects of the customer value chain.

2) Emotional Intelligence (EI), [28] [29] [30] [31] [32] [33], is about the capability of individuals or groups to recognize, assess, and manage their own emotions and emotions of others while planning their own behavior (also intuitive and creative) towards decisions and objectives; assuming also that such a capability can be enhanced by smart systems and devices that can capture, recognize, interpret, and process human emotions.

3) Business Intelligence (BI), [4] [5] [6] [7], is about automated analytical processing, knowledge discovery and visualization capabilities utilized on top of business data to support business-related decision-making and business discovery processes

EBI, in its current accentuation, is mainly about injecting the emotional theme common to the first two domains in order to augment the BI concept and practice. In essence, EBI envisions an emotionally sensitive, continuously learning business organization, as laid out in the next section.

\section{B. EBI model and value proposition}

EBI assumes the following working factors for its experience-centric, BI-based mission:

- Data (including customer sentiments) to propel it;

- Technology (emotionally sensitive ones) to assist and automate it;

- Business processes (with emotional components) to embed it;

- Governance (including emotional aspects) to steer it;

- Business value-creation (including the emotional one) for sustainability.

Dedicated developmental attention to each of these factors is pivotal although they cannot be separately discussed here.
Next, our vision of the EBI model is designed so as to explore and leverage experiential data from two main source realms: customers and organization (Fig. 1). Through its double-armed approach, EBI is conceived to equip business with an "emotion radar", decoding and linking capabilities on the customer analysis front, as well as supporting organizations to grow "emotion antennae" to sense emotive signals and collect wisdom from within. While the customer focus is mainly on external, quantitative data (e.g., channels and touch points, product interaction across key customer journeys), the organization focus is on internal, qualitative data, (e.g., employee knowledge crowdsourcing, HR).

Integrating these two insight sources, closed loop-type of 'knowledge discovery to improvement'-programs are foreseen to be developed to augment the incumbent BI-core. The main applied targets for industrialization are:

- EBI-driven Strategic Tracking and Decision Support (e.g., understand the drivers of good/bad experience. Quantify the Return-On-Investment for delighting a customer);

- EBI-driven Quality Assurance and Continuous Improvement (e.g., reveal broken experiences, identify disengaged employees and customers and prioritize actions to re-engage them);

- EBI-driven Product and Service Innovation (e.g., understand how to raise the level of customer intimacy in your innovation chain).

Not all of these EBI sub-programs need to be launched simultaneously, and for the most part they will also afford different types of data, technology, business processes, or governance models.

Technology will play a central role across all EBI programs, in Fig. 1 denoted as EBI Toolkit. Future business users and consumers need solutions that organize, interpret and visualize data-based insight in an easy-to-use manner for decision making. In our view, this would employ wide-spread concepts and practices such as Balanced Scorecards [34] and mobile dashboards, and new tools such as the FeelingsExplorer; all integrating organizational external and internal emotive information.

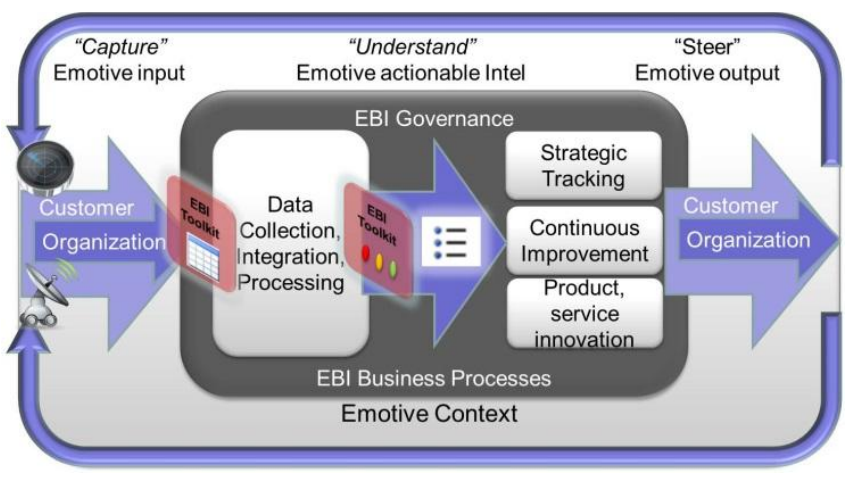

Figure 1. EBI Closed-loop Model: From Capturing via Understanding to Steering Emotions 


\section{THE FEELINGSEXPLORER - A BROWSER FOR EMOTIONAL BUSINESS INTELLIGENCE}

The FeelingsExplorer is an EBI technology prototype, operating as a kind of emotional mash-up browser, and allowing for emotion-aware decision support in different EBI applied contexts. It has been chosen as first technological "Proof of Concept"-target as it is able to demonstrate the management of emotive data and EBI ontology development in two business application areas that have a strong track record concerning a human-centric, experience-aware approach: Sales and Marketing as well as Product and Service R\&D.

As developmental frame served us the Global Understanding Environment (GUN), [35] [36], a vision of a ubiquitous, self-managed Semantic Web ecosystem where all kind of entities (Things, People, Abstractions) including their qualities can interact, serve, develop and learn from each other. According to the GUN vision, emotional qualities should be embedded directly to (or associated with) the products or services (or any other objects within the Internet of Things) enabling things to act autonomously (and interact with each other in machine-to-machine manner) constrained by highlevel policies and driven by personal decisions. This general vision, including the already developed GUN-based tools and technologies (e.g., UBIWARE [37] or the 4i technology, [38], allowing the visualization of objects' proximity in a semantic space) made GUN an ideal candidate for developing a first EBI technology, and the FeelingsExplorer served hereby as a fitting starting point and an inspirational showcase.

Conceptually, the FeelingsExplorer supports the process of ontology alignment (semantic mash-up) between a design domain ontology (design features of the products and services) and the customers' emotions domain ontology (part of the EBI ontology). The major pilot capabilities of the prototype are the management of emotional metadata and based on it: (a) the visualization of any product, service, or customer in a semantic space of both (design and emotional) ontologies; (b) the visualization of the semantic similarity of various products services and customers; (c) the visualization of personalized "emotional similarity" of various products, services, and customers (allowing a user to set up an personal emotional profile) [39]. Hence, the FeelingsExplorer can be seen as a semantic and machine-interpretable bridge between the human entity (business user or customer) and features of various business system deliverables (processes, products, and services) to facilitate business performance through emotional alignment.

\section{A. An Emotion-Driven Model of Decision Making}

Following our earlier argument about the inaptness and inferiority of purely ratio-driven thinking and action, we set out to formalize an emotion-dependent model of decision-making (see Fig. 2) (1).

$$
\begin{gathered}
F(\vec{p}, \vec{w})-\text { a decision-making function, where } \\
\vec{p}=p_{1}, p_{2}, \ldots, p_{n} \quad \begin{array}{c}
\text { - a vector of parameters/criteria for decision } \\
\text { making, }
\end{array} \\
\begin{array}{r}
\vec{w}=w_{1}, w_{2}, \ldots, w_{n} \text { - a vector of coefficients (measure of } \\
\text { significance) of criteria from vector } \vec{p} .
\end{array}
\end{gathered}
$$

Decision making and choice modelling are of course both tradition-rich fields of research with an impressive body of conceptual output, including the relevant focuses on business, artificial intelligence, and emotions [40] [41] [42]. For our purpose, a sufficient degree of model simplification was applied. When arbitrating between different options, humans operate on a set of available parameters/criteria that have an effect on their decision-making within a particular context. Existing experience and domain knowledge usually influence the development of idiosyncratic type and number of criteria, including transfer of emotional judgment, e.g., [43]. Another, important part is the valence or valuation of the criteria, which in our model is specified as vector of weights. This is a measure of the significance of each criterion for decisionmaking in a particular context and for a particular problem definition. Actually, this vector affects a number of criteria in vector $\vec{p}$ by simply assigning correspondent $w_{i}$ a value between 0 and 1 .

Context, of course, can influence vector $\vec{w}$ through various characteristics, including decision-making conditions, domain, person, and so forth. The list of such contextual components can be very extensive, and their utilization in the formal model depends on the ability to determine and capturing them. But, along with the more tangible contextual components, we need to deal also with emotional connotations that influence vector $\vec{w}$. In different emotional states, a person may generate very different vectors $\vec{w}$ (even when all other contextual constituents are kept equal). In the following, we focus on these emotional constituent and analyze its influence on decision-making.
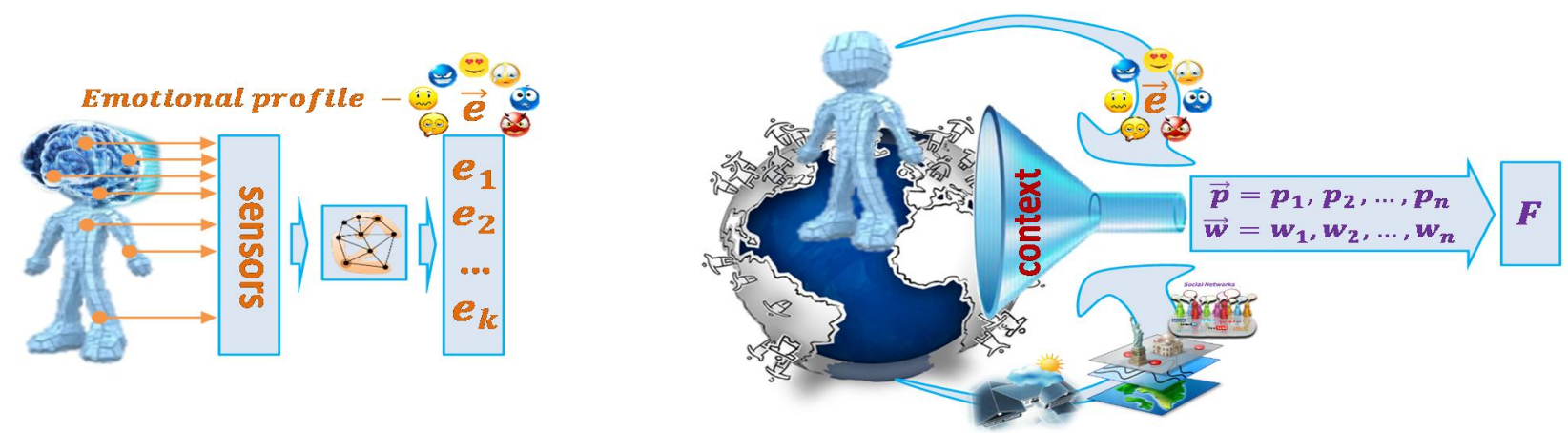

Figure 2. The Emotion-Driven Model of Decision Making 
For this we have separated the emotional part from the rest of the context and consider the vector $\overrightarrow{w_{0}}$ as a vector of weights formed under the influence of a context excluding emotional qualities. At the same time, we introduce vector $\vec{e}$, which captures a person's emotional profile parameters that influence $\overrightarrow{w_{0}}$, to finally result in vector $\vec{w}(2)$.

$$
\begin{aligned}
& F(\vec{p}, \vec{w})=F\left(\vec{p}, W\left(\overrightarrow{w_{0}}, \vec{e}\right)\right) \text { - a decision making } \\
& \text { function, where } \\
& W\left(\overrightarrow{w_{0}}, \vec{e}\right) \text { - a function that forms vector } \vec{w} \text { under influence of } \\
& \text { emotional profile, } \\
& \vec{e} \text { - a vector of parameters that form emotional profile of a person, } \\
& \overrightarrow{w_{0}} \text { - a vector } \vec{w} \text { formed under a context excluding emotional } \\
& \text { constituent. }
\end{aligned}
$$

From a practicality perspective, we are conscious that automated measurement and profiling of a person's emotional state is to date neither widely validated nor accepted. However, for our current purpose it is sufficient to note that the staggering development of sensor technologies enables, already today, the sensing of physical affect correlates. Such methods would allow us to roughly profile of a person $\vec{e}$ and then submit this for further research by psychologists to define a function $\Psi$ - a function dependent of vector $\vec{w}$ based on an emotional profile $\vec{e}(3)$. Hereby, we also assume the existence of methods and techniques to determine the correlation between an emotional profile and $\Delta \vec{w}$, that is the deviation of vector $\vec{w}$ from $\overrightarrow{w_{0}}$.

$$
F(\vec{p}, \vec{w})=F\left(\vec{p}, W\left(\overrightarrow{w_{0}}, \vec{e}\right)\right)=F\left(\vec{p},\left(\overrightarrow{w_{0}}+\Psi\left(\vec{e}, \overrightarrow{w_{0}}\right)\right)\right)
$$$$
\text { - a decision making function, where }
$$

$\Psi\left(\vec{e}, \overrightarrow{w_{0}}\right)=\Delta \vec{w}-$ a function of dependence of vector $\vec{w}$ from an emotional profile $\vec{e}$, where

$\Delta \vec{w}$ - a deviation of vector $\vec{w}$ from $\overrightarrow{w_{0}}$ under effect of an emotional context.

\section{B. EBI piloting scenarios of the FeelingsExplorer}

In the introduction to this section we have argued for the applicability of the decision-making model underlying the FeelingsExplorer to different targets of EBI practice; one obvious target being personalized sales and marketing. Predicting the decision-making function of a customer is a well-known technique widely used in advertisement for instance. Based on market research, business analysts build huge datasets of possible parameters/criteria $(\vec{p})$ and even their measure of significance $(\vec{w})$, in order to find an appropriate cluster for potential new customers, taking into account contextual information, while predicting vector $\vec{w}$ as average vector $\vec{w}^{\prime}$ of that cluster. Our proposed emotion-dependent model of decision-making can be considered as an enhancement approach to increase the precision of clustering and prediction of vector $\vec{w}$.

In doing so, we first separate/detach the emotional part of the context to get $\overrightarrow{w_{0}}$ ' for the cluster of individuals. This way we remove the inaccuracy (error) $\Delta \vec{w}$ that influences vector $\vec{w}$ for each member of the cluster. At the same time, the emotional profiles and correspondent $\Delta \vec{w}$ further help to build more precise clusters. As the most probable $\overrightarrow{w_{0}}$ for a new customer is derived, it becomes possible to model/simulate a set of vectors $\vec{w}$ in the context of different emotional profiles by adding correspondent deviations $\Delta \vec{w}$ to the vector $\overrightarrow{w_{0}}$. This provides us with an opportunity to create an emotional browser, that is the FeelingsExplorer, to preview possible decisions and actions of a person in an emotional space.

Thanks to the separation of the emotional constituent from the rest of the context and the collection of emotional profiles, it becomes possible to estimate an emotional profile of a potential new customer based on its clustering by other contextual parameters, in the case that it is impossible to capture the actual emotional profile. This offers even more flexibility for marketers to predict real needs of customers depending on the emotional profile of an event, purpose of purchasing, and so forth.

Another problem faced by market researchers is that previously made purchases and ordered services do not always correlate or reflect the actual needs and desires of a person. The fact that a person has bought mostly red-color and round-shape home design products does not necessarily mean that this is his or her favorite color or shape. Such purchases might be caused, for example, by preferences in interior design of the spouse, by availability, or by price. Or, the person may prefer actually very different products, when considering work surroundings. Even more pronounced is this conundrum in the case of designing new products, for which there are no exact market research object equivalents available, or which still offer an overwhelming degree of freedom in the hands of the creator. In all these cases, the actual challenge is to capture the emotional profile of the targeted person and the correct contextual information that influence decision making, something that can be supported with the FeelingsExplorer.

Taking into account also the customer as a direct user of FeelingsExplorer, our proposed model and emotional browser offers a very useful toolset to advise and facilitate decisionmaking for instance during purchasing tasks, either independently or in interaction with a sales representative. Purchase situations can be very emotionally-laden and stressful, and such conditions can lead a person to make (emotionally) inappropriate choices that later cause dissatisfaction, abandoning of a product or service, and even customer churn. Having available several products that meet a customer's need, the emotional browser can suggest the most suitable one, taking into account not only the "hot" emotional profile of the person, but a profile that she will have in most cases when using the product. At the same time, the user will have a possibility to browse her emotional space and see other corresponding suggestions of the browser in the context of other emotional profiles.

An even more practical use of FeelingsExplorer is when the purchase is for another person. For instance, in the case of buying a gift, it would be very useful and reasonable to see different options depending on the gift recipient's emotional profiles, to be able to effectively achieve a desired result. For example, this might be a case when a man would like to make an impression on a woman by surprising her with some jewelry. Assuming that the emotional profiles and initial vector $\overrightarrow{w_{0}}$ are shared by the woman and available to the emotional 
browser of the man, the browser can suggest several options for the gift. Let us imagine that mostly the woman prefers jewelry made of pearls and that the brand of jewelry is a second important criterion for her, while the size of the jewelry is among the less important parameters. However, the size of the jewelry may trigger the most intense positive emotional reaction, and this is exactly what our man is aiming for. Thus, pre-defining this desired emotional effect, the browser will recommend an abstract example of the product with correspondent properties. When connected to a database of real products (e.g., sponsored e-commerce), FeelingsExplorer can of course directly suggest concrete instances, closest to the abstract one.

We have built a prototype of the FeelingsExplorer as an extension to the previously developed $4 \mathrm{i}$ Browser [44] [38]- a smart visual context-sensitive resource browser. $4 \mathrm{i}$ is an ensemble of an Intelligent GUI Shell (smart middleware for a context-dependent combination of different MetaProviders) and MetaProviders, visualization modules that provide integration and context-dependent filtered representation of resource data.

The context-awareness and intelligence of its interface introduces a new feature that provides users with the possibility to receive not just raw data, but the required integrated information based on a specified context. Figure 3 illustrates the browsing of an emotional space with respect to the previously described jewelry gift example. Based on semantically-enhanced descriptions of emotional profiles, the number of contextual states and jewelry descriptions, one of the visualization modules of the 4 i-Browser presents us with a ranked set of products suitable for the specified purpose and emotional profile.

Through integration with other rapidly developing technologies, this type of emotion-dependent model can improve existent decision support techniques and approaches for various business stakeholders on both sides of the organizational perimeter. Obviously, the measurement of the emotional profile is still one of the major challenges. However, the Internet of Things, for instance, will in near future yield vast new possibilities for sensing and collecting all sorts of contextual data. This will also allow more accurate inferences for capturing the emotional profile of a person, through taking into account the location of the person, variety of things located nearby, objects that are in the focus of a person's view, and so forth.

This will further enlarge the space of use scenarios. For instance, the FeelingsExplorer could also be used in conjunction with customer voice feedback and monitoring methods, supporting the design and in-life management of products and touch points (e.g., by connecting different parameters of a customer service contact, including emotional characteristics of customer utterances), and to inform customer loyalty activities (e.g., by predicting customer engagement levels based on activity log patterns).

\section{SUMMARY}

Emotional Business Intelligence (EBI) has been exhibited as road to a future corporate BI that augments translating emotive information (e.g., customer sentiments) into businessrelevant decisions and action-allowing enterprises to step-up their emotional awareness and to turn this new intelligence into measurable business and brand value. Emotions, so one central argument, are vital for making truly smart and human-centric decisions, particularly in contexts of heightened complexity and uncertainty. This also applies to future dealing with Big Data; itself proposed as the silver path to future customer analysis.

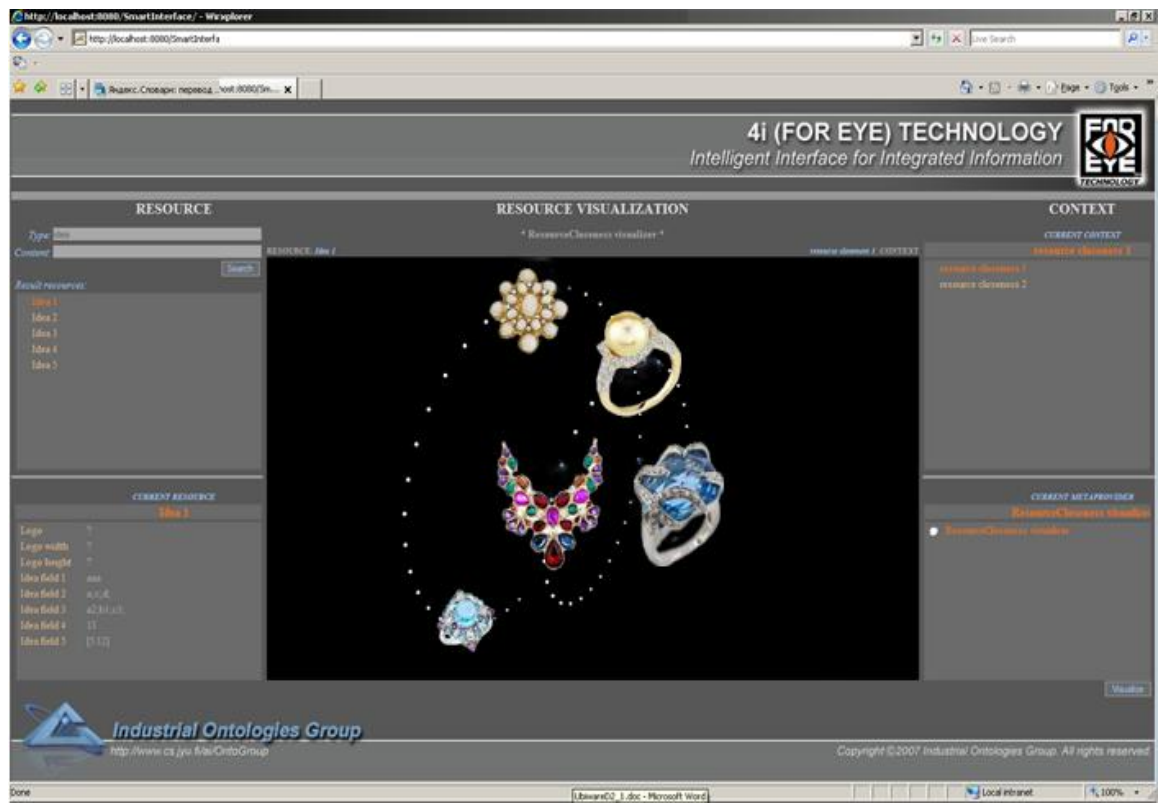

Figure 3. FeelingsExplorer Screenshot 
The conceptual framework for EBI was derived from amalgamating Emotional Business, Emotional Intelligence, and Business Intelligence to draft a working model of an emotionally smart, continuously learning business organization. It moves to action by sourcing customer- and organization-related emotive data, linking and mining them for meaning, and finally processing them into actionable insight. Three main underlying business paradigm premises set EBI apart from mainstream CX initiatives: (a) EBI must prove measurable business value, assuming that philanthropy and ethics, alone, are not sustainable grounds for investment; (b) EBI must be organizationally rooted and embedded through growing inclusion of employee experiences and transversal ownership; (c) EBI must be hard coded in business language through data, technology, processes, and governance.

In this context, a central role will also be assumed by technology that makes up the EBI Toolkit. In Section IV we portrayed the FeelingsExplorer to exemplify an implementation of an emotion-aware decision-making model, and its application to a handful of scenarios with business and customer users. The complete scope of the EBI Toolkit and service concepts, however, is foreseen to comprise a variety of solutions and software related to emotionally augmented data, their collection, processing, and presentation (i.e., EBI sensors, ontologies, algorithms, Scorecard, and Dashboard), EBI Key Performance Indicators, and an EBI organizational selfassessment tool, as well as an EBI training concept and service platform to deliver audit and transformation coaching.

\section{CONCLUSION}

Obviously, there remains much work to be done to further flesh out, concretize and validate the EBI in theory and practice. However, despite its imaginative nature the current report supports the advancement of different important evolutions.

For businesses it promotes the tangibilization and demystification of intangibles (e.g., experiences and emotions) as value drivers in enterprises. Business customers, partners, and one's own personnel make experiences, and they will define what products and services mean to them, how much they invest, and how long they stay loyal. So, the vital question is not whether experiences are business-relevant, but how to manage and potentially grow with and from them. EBI's mission is to equip business users with management-necessary means and insight. For marketers' and designers' usage, this was showcased by the FeelingsExplorer, enabling an advanced Kansei-type of engineering [17] [45].

Noteworthy is our firm assertion and value belief that "doing good" for employees and customers is not to be seen as a sustainable business objective in itself. In fact, experiencecentricity is too important to build its mission on ideological grounds alone. For this reason, EBI must pay back and deliver business value. While such financial implications are quickly proclaimed, actual cases and proof are still too rarely put forward. EBI's employment of data is therefore also aimed at measurable change and a quantifiable business case.

Big data- and evidence-driven business are contemporary, highly promising phenomena. In this vein, companies often sit on terabytes of customer data, of which its potential to drive an experience-centric business is not harnessed. Extra emotive data collection is not necessarily needed, but rather we need a new powerful concept and business model catalyst such as EBI that amplifies traditional BI into the central nervous system of an emotionally smart, continuously learning organization. EBI industrializes a quantified link between emotionally aware data and traditional business intelligence, helping enterprises to take this important and timely step today.

On the human (customer and user) in technological context level it seeks to stimulate new innovations that make sound use of rapidly evolving sensory technology and hereby gathers "living data". In combination with the Big Data trend described above we thus foresee two interdependent evolutions where human thinking, feeling and behavior is becoming increasingly accessible to automated measuring, quantification, and modelling; and where technology is becoming increasingly susceptible and sophisticated for integrating intuitive contents.

Therefore, in a philosophical dimension we see our research and the EBI concept also as promoters of voices that argue for closing the gap between computers and humans, via an emotional bridge (e.g., Ray Kurzweil's recent interview with www.wired.com), and in this context as renaissance of the pioneering visionaries' ideas on the role and meaning of technology for human enterprise [46] [47], EBI stipulates an important system intelligence transfer from human to business information processing.

\section{ACKNOWLEDGMENT}

We wish to kindly acknowledge the Industrial Ontologies Group's (www.mit.jyu.fi/ai/OntoGroup) developmental work regarding the GUN-based technology underlying the FeelingsExplorer; elaborated in the Finnish Agency for Innovation (Tekes) funded UBIWARE project.

We also thank Professor Vilma Luoma-aho and Professor Marita Vos for their conceptual contributions to EBI as regards to organizational drivers and conditions.

\section{REFERENCES}

[1] J.R. Bettman, "An Information Processing Theory of Consumer Choice", Reading, MA: Addison-Wesley, 1979.

[2] S.K. Card, T.P. Moran and A. Newell, "The Model Human Processor: An Engineering Model of Human Performance," in Handbook of Perception and Human Performance, Vol. 2: Cognitive Processes and Performance, K. R. Boff, L. Kaufman, and J. P. Thomas, Eds., 1986, pp. $1-35$.

[3] J. Lu; L. Niu; G. Zhang, "A Situation Retrieval Model for Cognitive Decision Support in Digital Business Ecosystems," Industrial Electronics, IEEE Transactions on , 60(3), 2013, pp.1059-1069.

[4] G. Nelson, "Business Intelligence 2.0: Are we there yet?," Proceedings of the SAS Global Forum 2010. Seattle, USA, April 2011, paper 0402010 .

[5] K. Schlegel, R. Sallam, D. Yuen, and J. Tapadinhas, "Gartner BI Magic Quadrant" 2013, February 2013. Available in: http://www.gartner.com/technology/reprints.do?id=11DZLPEP\&ct $=130207 \& \mathrm{st}=\mathrm{sb}$

[6] K. Carter, "Big Data: What's the Big Deal?," in: Think Business, NUS Business School online report, June 2013, Available in: http://thinkbusiness.nus.edu/articles/item/135-big-data-whats-the-bigdeal. 
[7] D. van Praet, "Unconscious Branding: How Neuroscience Can Empower (and Inspire) Marketing", Palgrave Macmillan Publ., 2012, p. 288.

[8] W. Edwards, "The theory of decision making," Psychological Bulletin, 51, 1954, pp. 380-417.

[9] M. Hassenzahl and N. Tractinsky, "User experience - a research agenda," Behaviour \& Information Technology, 25, 2, 2006, pp. 91-97.

[10] B. Lev, "Intangibles: Management, measurement and reporting," The Brookings Institution, 2001.

[11] M.B. Holbrook and E.C. Hirschman, "The experimental aspects of consumption: Consumer fantasies, feelings, and fun," The Journal of Consumer Research, 9, 2, 1982, pp. 132-140.

[12] B.J. Pine and J.H. Gilmore, "Welcome to the experience economy," Harvard Business Review, July-August 1998, pp. 97-105.

[13] J. Cross, "Emotional Business," Posted on February 3, 2013, Available in: http://www.internettime.com/2013/02/emotional-business/.

[14] R. Rao, "Emotional Business: Inspiring Human Connectedness to Grow Earnings and the Economy," iUniverse, 2012, p. 230.

[15] R. Verganti, "Design, meanings, and radical innovation: A metamodel and a research agenda," The Journal of Product Innovation Management, 25, 2008, pp. 436-456.

[16] S. Helfenstein, "Increasingly emotional design for growingly pragmatic users? A report from Finland ," Behaviour and Information Technology, 31, 2, 2012, pp. 185-204.

[17] A. Harada, "On the Definition of Kansei," in: Modeling the Evaluation Structure of Kansei, 1998, vol. 2, p. 22.

[18] E. Brunswik, "Wahrnehmung und Gegenstandswelt: Grundlegung einer Psychologie vom Gegenstand her", [Perception and the World of Objects. Foundation of a Psychology developed from the object]. Germany: Deutike, 1934.

[19] N. Bischof, "Das Rätsel Ödipus," [The Oedipus Riddle]. Germany: Piper, 1984.

[20] N. H. Frijda, "The Emotions," Cambridge University Press, 1986.

[21] D. Kahneman, D. and A. Tversky, "On the reality of cognitive illusions," Psychological Review, 103, 1986, pp. 582-591.

[22] V. Ermolayev, R. Akerkar, V. Terziyan, and M. Cochez, "Towards Evolving Knowledge Ecosystems for Big Data Understanding," in R. Akerkar, Ed., Big Data Computing, Chapter 1, Taylor \& Francis Group/CRC Press, November 2013, pp. 3-56.

[23] J. Fan, A. Kalyanpur, D.C. Gondek, and D.A. Ferrucci, "Automatic Knowledge Extraction from Documents," IBM Journal of Research and Development, vol. 56, no. 3/4, 2012, pp. 5:1-5:10.

[24] P. Livet, "Rational Choice, Neuroeconomy and Mixed Emotions," Philosophical Transactions of the Royal Society, London, (B: Biological Sciences), 365, 1538, 2010, pp. 259-269.

[25] A. Barnes, and P. Thagard, "Emotional Decisions," in: Proceedings of the Eighteenth Annual Conference of the Cognitive Science Society, University of California, July 1996, pp. 426-429.

[26] H. McCulloch, "A Model for Emotion-Based Decision Making (in an Era of Big Data)," Canadian Marketing Association, December 19, 2012, Available in: http://www.the-cma.org/about/blog/a-model-for-emotionbased-decision-making-in-an-era-of-big-data .

[27] S. Bucolo, and C. Wrigley, "Using a Design led Approach to Emotional Business Modeling," in E. Bohemia, J. Liedtka, and A. Rieple, Eds., Leading Innovation through Design: Pro-ceedings of the DMI 2012 International Research Conference, DMI. Boston, MA, USA, 2012, pp 323-333.

[28] D. Goleman, "Working with Emotional Intelligence," New York, Bantam Books, 1998.

[29] J. Mayer, P. Salovey, D. Caruso, and G. Sitarenios, "Emotional Intelligence as a Standard Intelligence," Emotion, 1, 2001, pp. 232-242.

[30] T. Bradberry, and J. Greaves, "Emotional Intelligence 2.0," San Diego, TalentSmart, 2009.

[31] R.W. Picard, E. Vyzas, and J. Healey, "Toward Machine Emotional Intelligence: Analysis of Affective Physiological State," IEEE
Transactions Pattern Analysis and Machine Intelligence, vol. 23, no. 10, October 2001, pp. 1175-1191.

[32] M. Minsky, "The Emotion Machine," Simon \& Schuster, 2006.

[33] R. Picard, "Affective Computing, " MIT Media Laboratory Perceptual Computing Section, Technical Report, 321, 1995.

[34] R.S. Kaplan and D.P. Norton, "The Balanced Scorecard: measures that drive performance," Harvard Business Review, Jan - Feb 1992, pp. 71 80 .

[35] V. Terziyan, "SmartResource - Proactive Self-Maintained Resources in Semantic Web: Les-sons Learned," International Journal of Smart Home, Special Issue on Future Generation Smart Space, vol.2, no. 2, April 2008, pp. 33-57.

[36] V. Terziyan, and A. Katasonov, "Global Understanding Environment: Applying Semantic and Agent Technologies to Industrial Automation," in: M. Lytras and P. Ordonez De Pablos, Eds., Emerging Topics and Technologies in Information Systems, Chapter III. IGI Global, 2009, pp. 55-87.

[37] A. Katasonov, O. Kaykova, O. Khriyenko, S. Nikitin, and V. Terziyan, "Smart Semantic Middleware for the Internet of Things," Proceedings of the 5-th International Conference on Informatics in Control, Automation and Robotics, May 2008, Funchal, Madeira, Portugal, pp. 169-178.

[38] O. Khriyenko, and V. Terziyan, "Similarity/Closeness-Based Resource Browser," in: J.J. Zhang, Ed., Proceedings of the Ninth IASTED International Conference on Visualization, Im-aging and Image Processing (VIIP-2009), July 2009, Cambridge, UK, ACTA Press, pp. 184-191.

[39] O. Khriyenko, V. Terziyan, and O. Kaikova, "End User-Assisted Interoperability in Internet of Things: Visually-Enriched User-Assisted Ontology Alignment," International Journal on Advances in Internet Technology, vol. 6, ns. 1\&2, 2013, pp. 90-100.

[40] M. Doumpos, and E. Grigoroudis, "Multicriteria Decision Aid and Artificial Intelligence," Whiley (UK), 2013

[41] D.E. Bell, H. Raiffa, and A. Tversky, "Decision making: Descriptive, normative, and prescriptive interactions," Cambridge University Press, 1988.

[42] J.D. Velásquez, "Modeling Emotion-Based Decision-Making," in: L.D. Cañamero, Ed., Emotional and Intelligent: The Tangled of Knot of Cognition, Papers from the 1998 AAAI Fall Symposium. Menlo Park, CA: AAAI Press, 1998, pp. 164-169.

[43] S. Helfenstein, "Product meaning, affective use evaluation, and transfer: a preliminary study," Human Technology, 1(1), 2005, pp. 76-100.

[44] O. Khriyenko, "Context-sensitive Visual Resource Browser," Proceedings of the IADIS International Conference on Computer Graphics and Visualization (CGV-2008), Amsterdam, The Netherlands, July 2008 .

[45] M. Nagamachi and A.M. Lokman, "Innovations of Kansei Engineering," CRC Press, September 2010, p. 152.

[46] J.C.R. Licklider, "Man-Computer Symbiosis," IRE Transactions on Human Factors in Electronics, vol. HFE-1, March 1960, pp. 4-11.

[47] D. C. Engelbart, "Augmenting Human Intellect: A Conceptual Framework," Summary Report AFOSR-3233, Menlo Park, CA: Stanford Research Institute, October 1962. 